\title{
2 The Cultural Organization of Attention
}

\section{PENELOPE BROWN}

\section{Introduction}

Language socialization is the process of socialization into language through language and its use in interaction (Schieffelin and Ochs 1986). Research in language socialization focuses on particular interactional practices in different cultural settings, asking how these proceed in situated interaction and how they influence the development of children's communicative skills and their ability to think, feel, and interact like others in their social world. Its unique contribution is the combination of detailed analysis of naturally occurring interactions and ethnographically sensitive interpretations of the presuppositions and understandings underpinning language practices that shape the child's understanding of taken-for-granted cultural truths.

Despite the proliferation of research in the language socialization paradigm over the last 30 years, relatively little has focused on interaction with prelinguistic infants. Yet, how new social members are drawn into the interactional practices of their society during their first year and a half of life is critical to understanding the biological bases, learning, and cross-cultural variability of social interaction as well as the role of culture more broadly in children's social-cognitive and language development (see also de León, this volume; Takada, this volume).

Language use rests on a bedrock of uniquely human competencies in social interaction, which unfold during the first year of life (Clark 2001). Humans appear to be biologically preprogrammed for collaborative interactional abilities in a number of respects, which collectively Levinson (2006) has dubbed the 'interactional engine.' These abilities relate to cooperation, intentionality, reading others' minds, coordinating attention, and establishing common ground (Clark 1996; Tomasello 2008). Human communication builds on these structures for collaborating, both evolutionarily and ontogenetically (Tomasello 2008).

The Handbook of Language Socialization, First Edition. Edited by Alessandro Duranti, Elinor Ochs, Bambi B. Schieffelin.

(C) 2011 Blackwell Publishing Ltd. Published 2011 by Blackwell Publishing Ltd. 
However, the evidence for universal underpinnings of interaction has to be reconciled with evidence for cultural specificity in interactional patterns, both in adult interaction and infant-caregiver interaction. This includes cultural differences in adult gaze patterns, conversational feedback mechanisms, and even in pointing behavior (e.g. Brown and Levinson 2005; Kita 2003, 2009; Rossano, Brown, and Levinson 2009). The anthropological and cross-cultural psychological literature on childhood provides abundant evidence for cultural variation in how infants are handled and socially engaged in their first year. Both the amount of interaction with infants and the features of the prelinguistic situation vary radically depending on social organization, household composition, socioeconomic activities of mothers and other caregivers, parental beliefs and cultural models, and ecological conditions - for example, mortality. These conditions influence the details of everyday experience for infants, from the physical arrangements of their handling (swaddling, feeding, degree of physical freedom) to the amount and nature of interactiveness: the positioning of babies as interlocutors whose 'utterances' are intentional communications, the amount of eye contact, turn-taking, and the kinds of participation structures into which an infant is drawn (de León 1998; see also de León, this volume). Interactional patterns are also influenced by adult beliefs about childhood and child rearing, including the contrast between child-centered versus situation-centered societies (Ochs and Schieffelin 1984) and, analogously, Lancy's (2008) distinction between gerontocracy (child-supported) and neontocracy (child-centered) societies.

Very little infancy research ${ }^{1}$ has examined the contextualized sequential details of infant-caregiver interactions during the first year of life. Modern theories of infant development (e.g. Bruner 1975a, 1975b, 1982; Elman et al. 1996; Masataka 2003b; Tomasello 1999, 2008) emphasize the influence of particular interactional practices in the child's developing communicative skills, claiming that the child's entry into social understanding is grounded in communication with others and that the extent and nature of social interaction a child experiences influence the development of his or her social understanding. But these theories have not taken sufficiently into account the implications of the fact that interactional practices with infants widely differ and are culturally shaped by beliefs about what infants need and what they can understand at different ages.

The current focus of infancy research on joint attention (see e.g. Moore and Dunham 1995) provides the basis for a set of predictions that can be fruitfully examined in cross-cultural interactional data. The critical age for coordinating attention in infancy - identified in the extensive developmental literature for infants in postindustrial societies - is between about 9 and 15 months, when major social-cognitive abilities emerge, including awareness of the other as an intentional agent and joint attention with a caregiver over a third object or event, referred to as the 'referential triangle' (Tomasello 1999). Around 12 months there is an important developmental milestone: babies look where adults are looking reliably, use adults as social reference points (gaze at them to check what to do in uncertain situations), act on objects like adults do, and actively direct adult attention through indicative gestures and pointing (Carpenter, Nagell, and Tomasello 1998). All of these are 
(putatively) essential prerequisites for coordinated interaction and later for learning language. The argument is that joint attention, arising out of social processes that are more basic than language, creates a base for referential communication.

We simply do not know, however, how culture-specific this story is. How is the interactional organization of joint attention socialized in infants in different cultures? The resources for drawing an interlocutor's attention everywhere include speech, gaze, body touching and postures, pointing gestures, and other actions, but it is well known that there are cross-cultural differences in adult deployment of these resources, ${ }^{2}$ so can we assume that they are deployed in comparable ways with infants everywhere? To answer these questions we need a more qualitative and comparative approach, one that can provide evidence of the process through which infants come to be able to coordinate attention in interaction in different cultural settings.

This chapter reports on recent steps in that direction, focussing on the prelinguistic period (to about 15 months of age) and on one type of cultural practice, the interactional organization of attention and how it is socialized in prelinguistic infants. The following discussion first sketches the developmentalist picture of infant social-communicative development in the first year, based largely on experimental studies in Europe, the United States, and Japan. This culminates in the 'nine-month revolution' during which several sociocognitive abilities come together as the infant comes to share attention jointly with others, as evidenced in pointing. The next section surveys recent research on how the coming-intojoint-attention process plays out in different cultural settings. The final section reports the author's findings on gaze and pointing behavior in infant-caregiver interaction in two nonindustrial societies, one in Mexico and one in Papua New Guinea, with radically different infant-caregiver interaction patterns.

Joint attention in prelinguistic infants has come into prominence as a research topic in the last two decades. Of course, the socialization of attention was a feature in the classic language socialization ethnographies (Kulick 1992; Ochs 1988; Schieffelin 1990), focussing on children beyond infancy. The socialization of attention goes on well beyond this early period, to be sure, sometimes with significant long-term effects (cf. Kulick 1992). ${ }^{3}$ But the focus in this chapter is the crucial developmental step around the end of the first year, when infants get an understanding of others as intentional agents who can direct their (the infant's) attention and whose attention can in turn be directed by themselves, so that they can jointly share communication about some specific object or event.

\section{Joint Attention in Infant-Caregiver Interaction in the First 12 Months}

\section{The developmentalist perspective}

Laboratory research aimed at understanding cognitive development and the cognitive prerequisites for language has shown that infants during their first year 
develop the ability to engage with others in joint attention. They do not start out with this ability. The picture based on research in postindustrial societies (mainly the United States, Europe, and Japan) is as follows: newborns spontaneously orient to human faces and imitate facial expressions, for example tongue protrusion (Meltzoff and Moore 1977); they are also sensitive to eye contact (Farroni et al. 2002). By two months they contingently respond to smiles and the gaze of an interlocutor (Bigelow and Rochat 2006; Murray and Trevarthen 1985). This disposition forms a basis for turn-taking: Masataka, for example, stresses the importance of sequentially dependent responding between Japanese caregivers and infants in social interaction, leading to conversational turn-taking as an early milestone, with coordination first of infants' suckling and mothers' jiggling behavior followed by coordination of vocalization and gaze (Masataka 2003b: 44). The details of mother-infant coordination have been shown to be culturally variable (Gratier 2003; Gratier and Trevarthan 2008), but it is generally accepted that some form of interactive coordination occurs (see also Takada, this volume). This interdependence relies on the mother's attribution of intentionality to the infant's vocalizations, and response contingency, features that are clearly evident in data of American and Japanese interactions with infants. In these contexts, the infant develops from spontaneously showing certain behaviors and expressive resources to exploiting these in interactive sequences as (s)he gains control over them while they are shaped in interactive routines with caregivers (Masataka 2003b). In these interactions, the adults credit the infant with social qualities and communicative intentions. This orientation, it is argued, is a crucial first step for the infant's development of intentional communication, and indeed for cultural variation in their communicative behavior (Masataka 2003b).

By four months, Japanese infants extend their index fingers (without, however, extending the whole arm). At this age these movements are related to exploring and self-regulation of attention, and the rate of doing this index finger extension correlates with infants' speech-like sounds. By five months, the infants share elaborate episodes of face-to-face engagement with their caregivers. These engagements suddenly become less frequent as the infant turns his or her attention from caregivers to objects. By six months the infant can follow the mother's gaze to an object if the object is in front of the infant and is the first object in sight as (s)he turns to look. Meanwhile, the development of intentional control over vocalization leads to babbling by about six to seven months.

It takes an infant several months to master the triadic relation of infantcaregiver-object communication. Pointing is seen as crucial for the development of referential communication, providing a nonverbal procedure for picking out a referent in the environment for oneself and another person to focus on (Masataka 2003b: 230-1; see also Bruner 1995). Interlocutors need to be in a context of joint attention in order to interpret gestures and other communicative acts. Establishing that an infant is jointly attending is not all that easy, and important evidence for common ground and mutual awareness of joint attention over a referent is the infant's 'gaze' from referent to interlocutor and back (Bates et al. 1979). The infant's gaze links together a referential act (pointing) and its meaning for an 
interlocutor ('now attend to THAT'). When infants can do this reliably, we can be sure that they have a referential understanding of the behavior of others and (in some sense) an understanding that others have minds and intentions like their own.

In sum, there is a developmental progression wherein babies become sensitive to an increasingly wide range of social signals between birth and nine months (Carpenter et al. 1998). From six to ten months babies show the beginning of clearly intentional behavior toward others. During the 9-15-month period they progress from sharing to following to actively directing another's attention. From 10 months on their first words start to develop. Around 12 months these early abilities come together in the developmental milestone that involves awareness of the other as an intentional agent and joint attention with a caregiver over a third object or event (the 'referential triangle'); that is, attending to the same thing but with awareness that each other is attending. Pointing is a clear indication of this achievement, including pointing for a range of different motives, not just imperatively ('I want that') but also declaratively ('Look! That's there'). Infants point to provide information for others, to point out new and absent referents (i.e. their pointing is referential), and to align and share attitudes (see e.g. Butterworth 2003; Carpenter et al. 1998; Liszkowski 2005, 2006; Liszkowski et al. 2004, 2009; Liszkowski, Carpenter, and Tomasello 2007, 2008; Masataka 2003a; Tomasello 1999; Tomasello et al. 2005; Tomasello, Carpenter, and Liszkowski 2007).

On this general picture developmentalists are in agreement, though there is much dispute over the details. ${ }^{4}$ Masataka (2003b: 241-2) summarizes the culmination of this developmental path in pointing and its significance for language as follows:

In order to comprehend the meaning of caregivers' acts of pointing appropriately, infants must coordinate their attention to both caregivers and objects and learn the communicative functions of referring or requesting. Otherwise, a singular focus would result in either interpersonal engagement (as infants attend to caregivers) or in severing the communicative channel (as infants attend to objects restrictively)... Only with the development of this ability do infants become able to understand the meaning of referential messages such as 'Look at this' and requests such as 'Give me that.' . . . infants begin to use gestures composed of manual movements and gaze patterns as well as speech-like vocalizations, to [express] communicative intentions such as requests for and reference to objects.

Cross-cultural developmental research in Africa has found that Yoruba infants (Trevarthen 1988) and infants of the !Kung San (Bakeman et al. 1990) follow the same developmental path. Masataka, however, suggests that, in societies or families where interlocutors do not point for infants, rather than an emphasis on referential speech it may be a more holistic kind of communication that promotes the child's entry into language - a clear prediction of possible cultural differences. ${ }^{5}$

\section{Implications of these processes for language acquisition}

The link to language is based on the idea that infant pointing is achieved by virtue of a particular response by the adult to the infant's pointing: labelling. Labelling 
the object being pointed at helps the infant to learn the word, but more importantly it leads to the understanding that others have communicative intentions (Masataka 2003b). A number of studies provide evidence that joint attention interactions do facilitate language learning (e.g. Carpenter et al. 1998; Tomasello and Farrar 1986; Tomasello and Todd 1983), showing clear links between joint attention and early vocabulary size, production of gestures, and length of conversations. This work rests on the early findings of Bruner and his colleagues, who argued that sociocommunicative routines scaffold the child's early language by providing him/her with an interpretable referential context via joint attention routines that help him/her to identify the adult's attentional focus and hence the intended referent (Bruner 1975a, 1975b, 1982, 1995; Ninio and Bruner 1978; see also Keenan and Schieffelin 1976). Brooks and Meltzoff (2008) also found a connection between infant gaze following and pointing and the infants' subsequent vocabulary development at age two. Childers, Vaughan, and Burquest (2007) reported on research in a rural community in Nigeria that showed that joint attention behavior among one-to-two-and-a-half-year-olds related to the development of both nouns and verbs, as reported by parents from a Child Developmental Index (CDI) checklist. In contrast, Masataka (2003b) found that the acquisition of the first five words among Japanese children is not correlated with the timing of the onset of pointing behavior, but the type of words in the vocabulary did correlate with pointing: common nouns (a positive correlation) and frozen phrases (a negative correlation). Kelly, Manning, and Rodak (2008) carry the argument about the relation between pointing gestures and speech into the child's second year, claiming that hand gestures significantly impact the brain's comprehension of speech and that the pointing gesture disambiguates indirect speech acts.

\section{Interactional Studies of Attention Management and Infant Pointing, 12-18 Months}

A related line of research focuses on joint attention in interaction in semi-natural but controlled 'free play' situations. Clark (2001) and Estigarribia and Clark (2007), for example, use such contexts to explore the establishment of 'common ground' (Clark 1996) and 'grounding' between American mothers and infants. Grounding, understood as 'the on-going process of establishing common ground in order to enable the joint projects of speaker and addressee in any exchange' (Clark 2001: 95), offers an 'opportunity space' for introducing new words and tracking the child's uptake of these new words. Clark (2001) analyzes grounding in adult speech to one-year-olds as they show unfamiliar objects, talk about them, and check on what the child means when their utterance is unclear. She finds that adults work to achieve joint attention, beginning with attention-getters (gaze, gestures, touch, attention calls, name). When the child is jointly attending, they use deictic terms to introduce words for new objects, using gesture and demonstration to maintain the child's attention (Clark 2001: 95). The joint attentional focus makes the connection between label and object obvious; its embedding in 
an interactive activity motivates the child to learn and remember the label. Estigarribia and Clark (2007) offer a model of how gaze and gesture contribute to joint attention in exchanges between American mothers and children, though they concede that this process is open to cultural variability.

Another line of research more compatible with a language socialization approach looks at joint-attention interactional sequences involving somewhat older children in naturally occurring settings. In an exemplary study from a conversation analytic perspective, Wootten (1997) analyzes his own English daughter's initial entry into requesting, from 12 months to three years. He shows the child's developing ability after age two to tailor the form of her requests to understandings about how events will unfold that have been established in the prior discourse, and hence how this one child 'enters culture.' This analysis probes the effects of momentary 'local' sequential understandings in interaction on the child's developing cognitive and social abilities. In a critique of developmental studies in the cognitivist tradition, Wootten suggests adding to the question of cognitive prerequisites these questions: what are the publicly available forms of action through which knowledge is expressed and how have those forms of action evolved? Addressing these questions, he argues, yields insights into how the child comes to understand the content of others' minds and how she comes to understand the world in (more or less) the same way others do.

In this spirit, Kidwell $(2003,2005,2009)$ and Kidwell and Zimmerman (2006, 2007) observed toddlers (age one to two and a half) in a large dataset of videotaped natural interaction in three Southern California daycare centers. In situations in which children were involved in sanctionable activities against another child (e.g. hitting, pushing, taking a toy away), these young children responded differently to types of caregiver gaze, differentiating 'the look' from 'a mere look' on the basis of their implications for whether or not the adult will intervene. The children adjust their conduct in relation to the caregiver's gaze, for example stopping or hiding sanctionable actions, indicating that one-to-two-year-olds are sensitive to features of the caregiver's gaze deployment - to its duration, fixation on a target, and relation to other activities of the caregiver. Kidwell and Zimmerman (2006) link these attention-organizing behaviors not just to the child's internal mental state or early understanding of intentionality (as developmentalists do). Rather, they argue that the children assess their own 'observability' via communicative resources that let the child 'read' the conduct of others and strategically adjust their own behavior. The early emergence of joint attention is one step on the way to a child's realization that their own actions can themselves be objects of attention.

Children's 'showing' actions are also sensitive to the activities of others (Kidwell and Zimmerman 2007). They position such actions at felicitous moments to get the attention of others and indicate their significance. Joint attention is fundamentally an interactional process, inseparable from the flow of social activity. 'Another's gaze shift constitutes a publicly available resource that offers participants opportunities to locate potentially relevant features of, and happenings in, the environment for their own attention and action' (Kidwell 2009: 148). Using the same 
daycare center data, Jones and Zimmerman (2003) argue that intentionality becomes visible in interaction between a 12-month-old child and a caregiver as the interaction unfolds. The child uses points and proto-words to orient to some feature of the environment in a way that makes a response by the caregiver relevant; the caregiver treats the child's behavior as intentional action directed to some end. Intentionality is thus jointly achieved by child and caregiver.

Studies of joint attention in a non-Western context look at the socialization of attention in a rather different way. Barbara Rogoff (Chavajay and Rogoff 1999; Rogoff et al. 1993) studied cultural patterns of attention management in San Pedro Guatemalan families and in US families in Salt Lake City, Utah, focussing on caregiver interactions with infants aged 14 to 20 months. Analyzing videotaped interactions in the home, they found that the Guatemalans were much more likely to attend to multiple events at once, keeping several attentional-interactional objects going simultaneously with a 'hummingbird' pattern of attention - with competing events smoothly attended to without the flow being interrupted. Salt Lake City attention patterns were much more single-focused. This pattern held both when the focus of attention was the toddler's activity and when it was adult interaction, suggesting that these are quite pervasive cultural practices. In short, the Guatemalans of San Pedro displayed a specific cultural preference in the deployment of attention in interaction. The authors suggest that the Guatemalans, with experience of many competing events in large households, have more practice in dividing their attention smoothly across multiple foci of attention. Attention to multiple foci are also facilitated by the Guatemalan cultural emphasis on keen observation as the basis for learning through 'intent participation' (Paradise and Rogoff 2009; Rogoff et al. 2003).

The big questions, of course, are these: does interactional variability across cultures matter developmentally? Can it influence the achievement of developmental milestones? The discussion below addresses this question through an ongoing comparative study conducted by the author on the integration of gaze and pointing in infant-caregiver interaction in two different social groups: the Tenejapa Tzeltal and the Rossel Islanders of Papua New Guinea (Brown 2007).

\section{Comparative Study of Caregiver-Infant Interaction}

\section{Caregiver-infant interaction in Mexico and Papua New Guinea}

This study addresses the question of whether the 'nine-month revolution' is affected by cultural differences in interactional style with infants. Video-recorded interactions between caregivers and 9-15-month-old infants in two nonindustrial societies were examined for evidence of infants' developing competence in engaging in joint attention episodes. One context is a Mayan society (Tzeltal) in Mexico, where interaction with infants during their first year is relatively minimal; the other is on Rossel Island (Papua New Guinea), where interaction with infants is 
characterized by intensive face-to-face communicative behaviors from shortly after the child's birth. Both societies are small-scale, rural communities based on kinship, with subsistence activities as the basic economic activity. Both are traditionalist and relatively isolated from the mainstream national culture, and the indigenous language prevails in the home (Tzeltal in the Mayan community; the Papuan language Yélî Dnye on Rossel Island). Inhabitants live in extended households: children have multiple caregivers (often child caregivers) and multiparty interactions are the norm, in contrast to the dyadic model familiar in studies of child-caregiver interaction in Anglo-American families. We are not, therefore, comparing an 'exotic' with a 'middle-class' society.

Differences in style of interaction with infants across these two speech communities are apparent even to a casual observer. Tzeltal infants are carried on the back in a shawl, are usually held, and are rarely set down for their first year. There are therefore strong physical restraints on their independent movement, and they have a restricted interactional space in which to operate. Living in small household compounds surrounded by fields, during their first year of life they have few interlocutors, and those they have tend to be relatively unresponsive to infants' preverbal 'utterances.' This is a society where interaction with infants is not a priority. Similarly to the Gusii of Kenya reported in LeVine et al. (1994), the chief goal of both mother and child caregivers is to soothe the infant and keep it calm, not to stimulate it. Adult interaction follows norms of restraint, nondemonstrativeness, and avoidance of eye contact, and this is also the case with infants; caregivers use body contact rather than social interaction to soothe. Adults do sometimes point for children, pointing out chickens and dogs, for example, but generally not with the aim of generating interactions but to distract the child or to instill fear as a basis for obedience.

By comparison, Rossel Island infants live in large hamlets of several households and spend a large proportion of time out of doors. They are carried in the arms or set down, or laid down to sleep, and they usually have a large public space to move around in and explore. They often have many interlocutors, including passersby through the village, who greet them and are generally responsive to infants' 'utterances.' In short, Rossel Islanders surround infants with interlocutors who actively engage them and are responsive to their 'utterances,' similarly to AngloAmerican middle-class child-centered norms. ${ }^{6}$

Do these differences influence how infants learn to coordinate attention in interaction, and, ultimately, their pragmatic and linguistic development? During fieldwork over a number of years, I have observed social interactions with prelinguistic infants in both societies. The methods used are broad-ranging, including participant observation, video-recording of naturally occurring caregiver-child interaction, parental interviews, and systematic sampling of behavior and interaction with infants. The corpus contains 69 hours of video recordings of interactions with 33 Tzeltal infants and 73 hours with 44 Rossel infants in the age range up to 18 months, as well as many of their older siblings and cousins. Longitudinal samples for five Tzeltal children and six Rossel children were also collected across different contexts and over up to several years (I have used different subsets of 
this data in what is reported here). The overall aim of the project is to combine quantitative measures to probe the observed contrasts in amount and style of infant-caregiver interaction with ethnographic and conversation-analytic methods to examine the kinds of socialization for communication that occurs, the normal patterns of daily life affecting infants in the two societies, and cultural beliefs about children and parenting.

The analysis below focuses on the interactional management of joint attention in the 9-15-month period, including episodes of gaze following, index-finger point following and production, and the integration of gaze and vocalization with pointing. It asks whether there is any evidence that the different interaction practices have differential consequences in the children's development of communicative understanding. ${ }^{7}$

\section{Baseline for infant activity: Five-minute samples}

To establish a baseline of infant interaction, one-to-two-and-a-half-hour stretches of naturally occurring interactions of nine Rossel Island infants (five boys and four girls) and eight Tzeltal infants (four boys and four girls), either directly observed or in video recordings, were sampled at five-minute intervals, producing 'snapshots' of infant activity at different times of the day. The children were at comparable developmental stages and alert and available for interaction. The data for this measure are summarized in Table 2.1.

The kinds of activities the infants were engaged in during each snapshot judged as their primary focus of attention at the instant sampled ${ }^{8}-$ were coded as follows:

- BF: Attending to bodily functions (sleeping, eating, suckling, being clothed, being bathed, etc.).

- SO: socially oriented; interacting with other(s).

- PwO: playing with or manipulating an object by oneself.

- S-A: self-absorbed; doing nothing beyond looking around, moving around; just 'being.'

- CT: can't tell; for example, child not visible on camera.

These very general codes exhaustively categorize the activities captured in the five-minute sample 'snapshots.'

Table 2.1 Data for five-minute samples

\begin{tabular}{lccc}
\hline & $\begin{array}{l}\text { Number of } \\
\text { Infants }\end{array}$ & $\begin{array}{l}\text { Total Hours } \\
\text { Sampled }\end{array}$ & $\begin{array}{l}\text { Number of Datapoints (Sampled } \\
\text { Every Five Minutes) }\end{array}$ \\
\hline Rossel & 9 & 38 & 482 \\
Tzeltal & 8 & 22 & 272 \\
\hline
\end{tabular}




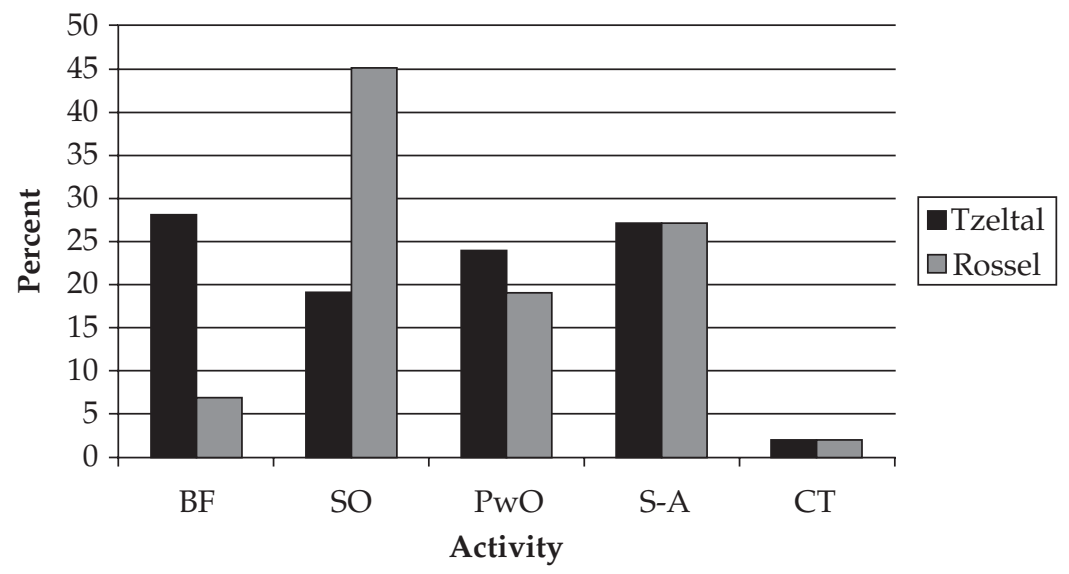

Figure 2.1 Results, five-minute samples, Tzeltal versus Rossel.

Figure 2.1 displays the relative frequencies of these activity types for Rossel and Tzeltal infants. There was a clear and dramatic difference in the two populations in the proportion of samples in which the infant was 'socially oriented' (primarily focussed on interacting or attempting to interact with someone) (Rossel 45 percent, Tzeltal 19 percent). The Tzeltal infants were much more often engaged in 'bodily functions' (eating, suckling, sleeping), although this is possibly an artifact of sampling times. There were no differences in the frequency of Rossel and Tzeltal infants playing with objects or being self-absorbed (doing nothing beyond observing the world). ${ }^{9}$

\section{Interactional density}

Several hours of videotaped data for these same infants were further analyzed for 'interactional density,' or the amount of interaction per unit time. Because measuring the density of interaction is fraught with difficulties, analysis focused on initiation of interactional sequences, where one participant makes an initiating move, a 'summons' to interaction, which is not necessarily responded to. The initiation of a 'sequence' was defined as a new focus of attention or new addressee about it, or new propositional content or attitude expressed to it. ${ }^{10}$ In the discussion that follows we examine initiations in interactions involving two Rossel infants (aged 10-11 months) and three Tzeltal infants (aged 11-12 months).

The results of this measure are summarized in Table 2.2, which shows that there were twice as many interaction initiators per minute in the Rossel samples, compared with the Tzeltal ones.

A second question immediately arises: who is actually doing the initiating, the infant or the interlocutor? Table 2.3 shows that the Rossel and Tzeltal infants do not dramatically differ in the frequency with which they initiate interaction with 
Table 2.2 Interaction initiators (per minute)

\begin{tabular}{lcccc}
\hline & Age Range & $\begin{array}{l}\text { Number of } \\
\text { Interaction } \\
\text { Initiators in } \\
\text { Samples }\end{array}$ & $\begin{array}{l}\text { Range of } \\
\text { Interaction } \\
\text { Initiators } \\
\text { per Minute }\end{array}$ & $\begin{array}{l}\text { Mean Number } \\
\text { of Interaction } \\
\text { Initiators per } \\
\text { Minute }\end{array}$ \\
\hline $\begin{array}{c}\text { Tzeltal (3 children, } \\
\begin{array}{c}3.5 \text { hours) } \\
\text { Rossel (2 children, } \\
2 \text { hours) }\end{array}\end{array}$ & $11-12$ months & 478 & $1.6-5.9$ & 3.4 \\
\hline
\end{tabular}

* More data for the Tzeltal infants was needed, to obtain a comparable number of interaction initiations.

Table 2.3 Infant- versus other-initiated interaction initiators

\begin{tabular}{lll}
\hline & Tzeltal & Rossel \\
\hline Infant-initiated & 1.9 per minute & 2.3 per minute \\
Other-initiated & 1.5 per minute & 5.1 per minute \\
\hline
\end{tabular}

another person - 1.9 initiating moves per minute for the Tzeltal infants versus 2.3 per minute on average for Rossel. But Rossel caregivers initiate interaction with infants (in these samples) more than three times as often (per minute) as Tzeltal caregivers do! In other words, Rossel infants are interacting a lot more largely by virtue of the fact that others frequently initiate interaction with the infant. This finding supports the ethnographic observations of Rossels actively trying to interact with babies and the Tzeltal being much more restrained, waiting till the infant is ready and then responding to the infant's own initiatives.

The implications of this finding are potentially consequential, given that the 'referential triangle' development (Tomasello 1999) in the 9-15-months age range critically depends on interaction patterns between infants and caregivers. Do Rossel infants display understanding of the referential triangle earlier than Tzeltal infants? To answer this, we need a second line of inquiry: analyses of the emergence of Rossel and Tzeltal infants initiating joint attention with another over an object/event, and an assessment of at what age this behavior begins.

\section{Joint attention episodes in Rossel and Tzeltal}

Joint attention episodes, where the infant is trying to get someone's attention or someone else is trying to get the infant's attention, were coded in the videotaped 
data for six types of attention-sharing behaviors, ${ }^{11}$ which in laboratory studies have been found to be involved in the developmental trajectory to joint attention:

- Pointing: trying to get someone's attention or share attention through pointing at an object or event.

- Reaching: holding hand out towards object and signaling (vocalization, gaze) to interlocutor.

- Indicative gesturing: gesturing towards object and signaling to interlocutor.

- Showing: holding out an object to interlocutor.

- Vocalizing: attention-getting sounds or speech.

- Gazing: mutual gaze, versus infant-interlocutor gaze, at indicated object/ event.

Four questions guided the analysis of gaze-pointing attention management actions (Liszkowski and Brown 2007):

- Do caregivers point for 9-15-month-old infants in both societies?

- Does pointing have the canonical index-finger-extended form?

- Do 9-15-month-old infants follow others' points/gestures that aim to draw them into joint attention at this age in both societies?

- Do infants themselves initiate joint attention by pointing/gesturing at objects at this age in both societies?

The answer is 'yes' to all four questions! At least some Rossel and Tzeltal infants do point for joint attention, canonically with outstretched arm and extended index finger, some of the time. The following examples of index-finger pointing by adults and by infants illustrate how these interactions unfold in the two cultural settings.

\section{Example 2.1: Rossel [2003v10]}

Participants: Dini (D, 14 months) and his Uncle (Unc), his aunt (Aun), and Maria (Mar, D's teenage caregiver), with various other children off camera. The adults are sitting around chewing betelnut and relaxing near the river.

$\mathrm{D}$, standing right next to his uncle's back, squats and urinates on the ground. Maria (off camera) calls attention to D's action, laughing and calling out, as Uncle looks down at D:

Mar: kââ vye pwo paa? hehehe

'Is urine out up going?' ((laughs))

Aun: ((turns and looks, then says excitedly))

Ee Dinimgaa dê vy:oo!

'Eh, Dinimgaa has peed!' 
Unc: ((taps D on the shoulder and says 'huh' several times, until D gazes at him, then he (Unc) points at the puddle))

Kê n:uu? (0.9) Kê n:uu?

'This who (did it)? This who?' ((pointing at puddle))

D: $\quad(($ squats and looks down at his puddle))

Unc: N:uu dê yo?

'Who did that?' ((pointing))

(0.9)

kê n:uu? (1.3) kê n:uu?

'This who? This who?' ((pointing))

D: $\quad($ turns and walks away))

Here several participants draw attention to the child's delict, and no further sanction ensues. Notice that there is more to joint attention than just pointing to an object to identify it: the adult points to the result of an action (puddle), indirect evidence of the child's delict (urinating on the ground next to his uncle). The accusation is also indirect ('Who (did) this?'). Dini responds to his uncle's summons by gazing at him, and then he gazes at the puddle. He does not point at it but shares his uncle's attention and (potentially) the message that his uncle does not approve (see Figure 2.2).

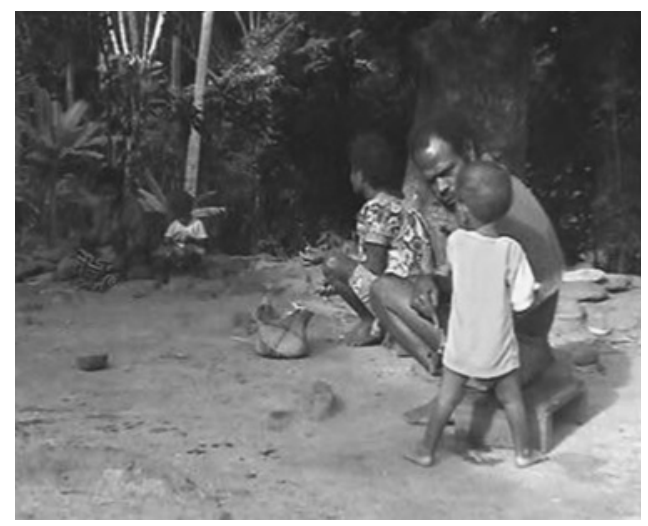

Figure 2.2 Dini, his uncle, and his puddle of urine.

Example 2.2 illustrates a Rossel child drawing others' attention and sharing excitement over the jointly-attended-to referent.

\section{Example 2.2: Rossel [2005v23]}

Participants: N:iin:ii (N, 10 months) with his mother (Mo), the researcher (PB), N's six-year-old brother (Br), and his uncle (Gh).

Mother and N:iin:ii are sitting together on their house verandah, with $\mathrm{PB}$ and Br standing at the foot of the steps about eight feet away. N:iin:ii's 
attention turns to his uncle kicking a ball about 30 feet away. N:iin:ii squeals (nonsense syllables of excitement; he can't yet produce recognizable words):

(// indicates speech or behavior overlapping with prior turn; = indicates latched speech)

$\mathrm{N}$ : gu ii! ikee! ((turns gaze to PB, back to uncle/ball, and points)) iye. ee. EEEEEEE! ((affect and gaze toward soccer ball))

Mo: ((gazes at $\mathrm{N}$, then at ball)) ii. ball! // ball! ii?= 'Ii. Ball! Ball! Ii?'12

$\mathrm{N}: \quad / /$ ee.

Mo: = ii. ball ball. ball hii. . . ii ball ball ball (ndêwe).= 'Ii. Ball ball. Ball. Hii . . . ii ball ball ball (ndêwe).'

$\mathrm{N}$ : / / eе eе eе eе

Mo: $=i i !$

'Ii!'

Gh: ((calling from afar)) a nu a nu a nu!

'To me to me to me! ${ }^{13}$

Mo: hii! =

'Hii!'

$=($ (points) $)$ soccer soccer soccer. soccer.

'Soccer soccer soccer. Soccer.'

soccer, ehe.h hii hii. hii hii hii hii.

'Soccer., eheh, hii hii. hii hii hii hii.'

$\mathrm{N}: \quad / /(($ gaze turns to $\mathrm{PB} / \mathrm{Br}$, then back))

Gh: $\quad / /(. . .$.

'[unintelligible]'

((Mo helps $\mathrm{N}$ to stand up, holding onto his hand))

$\mathrm{N}$ : ee

Gh: // soccer! soccer!

'Soccer. Soccer!'

$\mathrm{N}$ : ((gazes at $\mathrm{Gh} /$ ball, struggles to climb down from porch, Mo restrains him))

Mo: // he. he he he. He used to go down.

'Ehe. Hii. He used to go down.' ((to PB, referring to N's attempt to get off porch))

hm. hm. hm. soccer.

'Hm. Hm. Hm. Soccer.'

$\mathrm{N}$ : ee ((waving hand gesture towards Gh/ball)) EEEEEEEEE!

Mo: ehe. ii. ii. soccer.

'Ehe. Ii. Ii. Soccer.'

$\mathrm{N}$ : ee ide. de de ((pointing to Gh/ball))

Mo: / / Ide de de de de de de de [nonsense syllables] ((pointing at uncle/ ball, alternating gaze between N's face and Gh/ball )) 


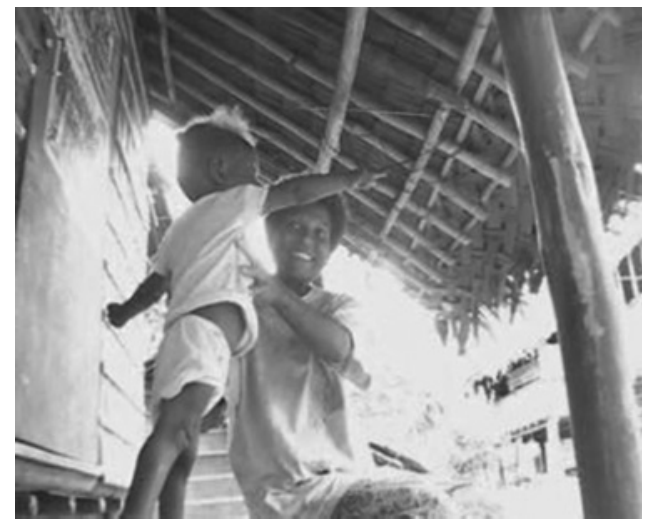

Figure 2.3 N:iin:ii and mother, with uncle, ball, and bystanders.

The name of the game here is sharing attention and affect over an object/event in a proto-conversation, exchanging excited and largely meaningless cries about playing ball (see Figure 2.3). The mother labels the event (calling it 'soccer') as well as the 'ball,' and takes an encouraging, facilitating role in the child's expression of excitement. Others (PB, Gh) are drawn into the interaction via the child's gaze and vocalizations; the result is collaborative, often simultaneous expression of excitement during joint attention, culminating in $\mathrm{N}$ and Mo jointly pointing.

In both these Rossel examples, interlocutors widely dispersed in space (up to 30 feet away) coordinate to share attention with the infant. While Tzeltal interactions are much more spatially constrained, analogous instances of joint attention are enacted, as illustrated below. In Example 2.3, Lus points for her father to draw an object into joint attention.

\section{Example 2.3: Tzeltal [2005v5B]}

Participants: Lus (12 months) and her father (Fa).

The whole family (both parents, four children) is sitting around relaxing in the cooking hut. Here the father's gaze is on the infant, who has his attention.

Lus: ((looks up to clothesline, points at her pants hanging on the line))

Eee.

$(($ looks at $\mathrm{Fa}))$

Fa: ((gazes to referent))

ba'ay?

'Where is it?'

Lus: eee ((gazes at Fa, while point is held out at referent))

Fa: eh in nix yael a, a'pantalon.

'Oh just look there ((gesturing to referent)), your pants.'

((The episode ends as Lus's attention shifts to a toy car on the ground.)) 


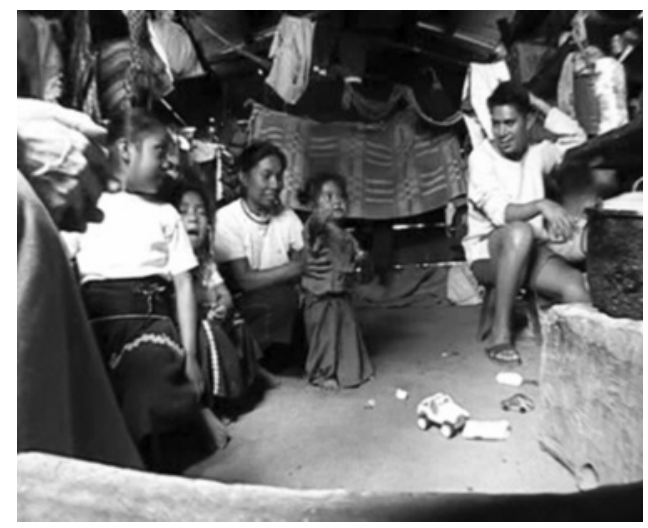

Figure 2.4 Baby Lus, father, and pants.

This Tzeltal example is a particularly clear case of a 'referential triangle episode': index-finger pointing along with gaze-checking by the infant, and a response that turns attention to the referent and labels it for the child. Note, however, that the father's first response is not a label but the question banti ('where?'), a standard response to infant pointing in Tzeltal. And the episode is brief and self-contained, unconnected to what precedes and what follows it (see Figure 2.4).

Example 2.4 shows a more extended exchange over an object being jointly attended to.

\section{Example 2.4: Tzeltal [2006 v26]}

Participants: Xmik (Xm, 12 months) and her mother (Mo).

Mother and infant are inside the house, Mother sitting on a chair, baby Xmik sitting on the floor facing away from Mo. Xmik initiates joint attention to a pet bird, and a proto-conversation ensues:

Xm: ((looking around, her attention comes to focus on the bird hopping across the floor))

hm. ((pointing at bird))

Mo hm.

'Hm.'

Xm: ((looks at Mo))

hm. ((gesturing at bird))

Mo: / / la' me uta.

'Come here, say to it'

la' me uta.

'Come here, say to it.' 
$\mathrm{Xm}$ : me ((looking back at bird and gesturing toward it))

Mo: hm. ila'wil.

'Hm. Look.'

$\mathrm{Xm}: \quad a^{\prime}$ me ((gesturing to bird))

Mo: Hm.

Xm: ((turns to look at Mo))

((a few moments later, Mother re-initiates attention to bird)):

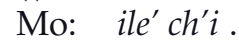

'Here it is for sure.'

((points over her shoulder to bird))

Xm: ((turns and gazes at bird))

Mo: ((gazes at Xmik))

$w a^{\prime} y$.

'You see.'

$\mathrm{Xm}$ : hee ((pointing to bird))

Mo: in.

'This one.'

Xm: ((looks away))

Mo: in te lumine. ((points over her shoulder again, looks at bird, then back at Xmik))

Xm: ((gazes where Mo points, then shifts gaze and points to where bird hee. has now gone))

Mo: in. li' bajt li'i. ((pointing to floor, where bird has now hopped to)) 'Hm. Here it went here'.

Xm: $h m$ ((pointing))

Mo: hm

Here Xmik calls her mother's attention to the bird and her mother responds noncommitally until Xmik looks around at her, then suggests what she (Xmik) should say to the bird ('Come here, say to it'). Twice Xmik partially repeats the instructed words ('me,' 'a me'), and Mo's response is minimal ('Hm.' 'Look'). For the most part, the infant's gaze tracks the object, not the interlocutor's attention; she is presuming (or possibly indifferent to) the mother's visual attentional focus. At no point is the name of the bird uttered, and the mother does not treat this as an opportunity to teach the child words (see Figure 2.5).

In general, Rossel and Tzeltal infants' pointing appears within the expected age range of 9 to 15 months, though it is not found in the sampled data for all the infants. ${ }^{14}$ In both societies babies point at objects (a bird, a ball, a piece of clothing) and adults point both at static objects (toys, animals, people) and at events (Dini's urine on the ground, piglets coming across the field, the rain starting to fall). Jointattention caregiver-infant episodes in Rossel are more frequent and they tend to 


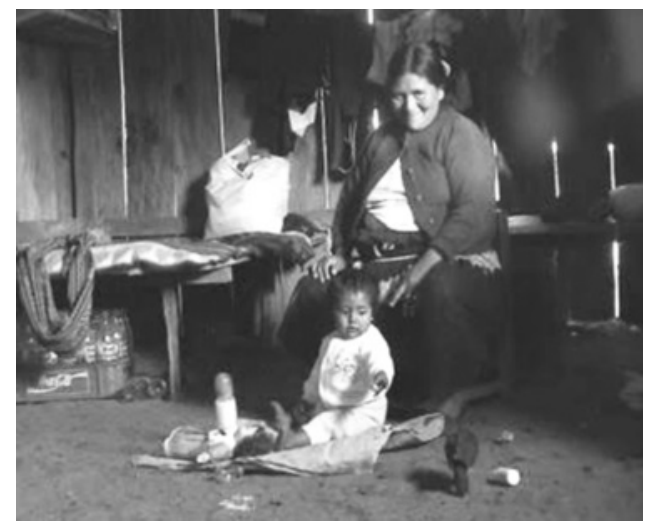

Figure 2.5 Baby Xmik, mother, and bird.

be longer and more affectively aroused than in the Tzeltal community; however, they are similar in this fundamental respect: joint attention is clearly achieved and the child displays a sense of the others' communicative intention to share attention over an object or event. ${ }^{15}$

These findings support developmentalists' claims (e.g. Butterworth 2003) that infant pointing appears around 11 months, suggesting a biological basis to pointing as a joint-attention behavior. However, at this age, spontaneous pointing by Tzeltal and Rossel infants is rare and not evidenced in the data samples for all infants, and the infants are not yet very competent at pointing: they often do not attend to others' points, often do not cue what they are pointing at with their gaze, and do not reliably gaze to check the affective response of the addressee. Pointing is just one of several ways - holding out an object, reaching or gesturing towards things, vocalizing, gazing - of bringing something into joint attention, and all the infants show evidence of achieving joint attention by one or more of these means. In both the Tzeltal and the Rossel data, these other forms of initiating joint attention predominate. In addition, pointing in Tzeltal and Rossel joint attention interactions with children does not have the canonical result observed in postindustrial societies, with the adult labelling the object pointed at. Indeed, in Tzeltal, caregivers' responses do not usually label the object but instead acknowledge it (e.g. 'where is it?') or attempt to get the child to interact with it ('tell it to come here'). Tzeltal infants' gaze patterns in these episodes reveal a presumption of (or indifference to) interlocutors' attention.

On the basis of these observations, it is hard to believe that indexical pointing per se is playing a critical role in the infants' understanding that others have minds and communicative intentions of their own. Other forms of drawing an interlocutor into joint attention do not seem to be sharply differentiated yet from pointing, and nonetheless both Rossel and Tzeltal infants clearly display attention to others' communicative intents, and in turn draw others' attention to a joint focus. 
The research reported here is ongoing. Analysis has not yet determined whether there are demonstrable differences at this age between Tzeltal and Rossel infants' gaze behavior in joint-attention episodes, or in how these episodes sequentially unfold. What is clear is that, despite the differences in interactional style between Tzeltal and Rossel, there is no evidence so far that the highly active style of Rossel infant-caregiver interaction brings in the referential triangle earlier for Rossel than for Tzeltal infants.

\section{Conclusions}

The results from this study are compatible with the view of developmentalists that the cultural organization of attention operates on a preprogrammed biological base. Infants have proclivities to share attention and their abilities to do so blossom in the period of 9-15 months. Infants are not all little clones - there is considerable individual variation, and likely cultural variation in the interactional sequential details, if not of the developmental sequence, of joint-attention behavior. Tzeltal caregivers are much less interventionist and less affectively expressive in interacting with their infants than are Rossel Islanders, yet these differences do not seem to have a radical effect on the emergence of pointing. While the interactions are socially and culturally organized and reveal what caregivers take to be interesting objects for children's attention, the desire for interaction over objects between infants of this age and their caregivers is apparent in both locales.

An analogous conclusion was drawn by the infant specialist Barry Brazelton, who studied Zinacanteco Mayan babies. Despite dramatic differences in the amount of stimulation provided by Mayan mothers (in comparison with mothers in the United States), Brazelton (1977) found that Zinacanteco and American babies develop at a comparable rate. In the absence of contingent reinforcement for smiling, vocalizing, and motor development, Mayan infants walk, can be coaxed to smile and vocalize, and appear to speak on time. Brazelton (1977: 177) proposes that developmental milestones are not notably affected by these kinds of differential interactional treatment in infancy. A similar point is made by Schieffelin and Ochs (1983) with respect to the absence of Baby Talk. Yet this issue remains controversial: others have argued (for other populations) that there are clear differences in development linked to differential conditions in infancy (see e.g. Shweder et al. 2006).

Can we generalize to universals in interaction with infants? Many authors have argued for the universality of certain acoustic/prosodic features of child-directed speech (e.g. Fernald 1992a, 1992b; Kuhl et al. 1997; Monnot 1999). Harkness and Super (1996: 2) offer two-year-old tantrums as a candidate universal. Lancy (1996: 83) suggests a universal tendency for infants to observe and imitate elders. Playing with objects is another candidate (Lancy 2008: 159); where children lack toys they pick up random objects (sticks, stones, flowers) to play with. We propose index- 
finger pointing at around 12 months as a candidate universal, one that is currently being explored in the Communication before Language project at the Max Planck Institute for Psycholinguistics. Evidence for pointing as a universal is that a critical age for joint attention is found in pointing behavior, which appears within the same age range in widely varying cultures (Liszkowski et al. forthcoming). Universals are arguably more likely to be found in these fundamental underpinnings to communicative interaction - in attention management or turn-taking, for example - than in the details of language structure (Evans and Levinson 2009; Stivers et al. 2009).

What then are the implications of cultural differences in social interaction with prelinguistic infants? While the imputation of intentions to infants' behavior may be a universal, the disposition to do so varies radically across situations and social groups, and intentions imputed do not necessarily lead to social interaction. Kulick (1992: 100) reports that New Guinea Gapun caregivers impute intentions to infants, but these are most often aggression, anger, or dissatisfaction, and lead neither to labelling nor to positive interactions with the infant. In addition, the mere fact that infants point does not necessarily mean that pointing leads to joint attention and an understanding of others' communicative intents; the critical developmental modality may well be something more diffuse such as gestural indicating, not explicitly index-finger pointing. Social groups may also differ in the kinds of objects to which infants' attention is directed and the social ends of such acts. Gapun caregivers, for example, point for infants to focus their attention on something outside of themselves, but the thing pointed at is often not actually there! Until the child itself initiates these routines (Kulick 1992: 121),

it must often be unclear what a prompt to look at actually refers to, since the referent will either be obscure (as when a mother points towards a mass of trees in the distance and tells her child ... 'There a bird'), invisible (as when children are told to look at spirits whom caregivers claim are coming to get them), or something completely different from what is being pointed at.

Similarly, Tzeltal pointing for infants is frequently a matter of pointing at things that are not clearly discriminable. This practice, along with the absence of labelling, suggest that the link between joint attention, pointing, labelling, and the child's understanding of referential actions is not necessarily as straightforward as it would appear from studies of infant interactions in university laboratories elsewhere in the world. This raises the possibility that, in the cultural organization of attention, indexical pointing does not necessarily play a special role.

A comparative perspective on social practices in caregiver-infant interactions will allow us to refine our understanding of the role of joint attention in learning to become a communicative partner. Children are socialized through and into different interactional styles across different cultural settings, yet the evidence so far supports the view that there is a universal propensity for children to engage in episodes of joint attention over objects and events by around the age of 12 months. 


\section{NOTES}

1 For overviews of the comparative infancy and early childhood literature from a variety of disciplinary perspectives, see, for example, Bullowa 1979; Eibl-Eibesfeld 1983; Field et al. 1981; Harkness and Super 1996; Jahoda and Lewis 1988; Lancy 2008; Leiderman, Tulkin, and Rosenfeld 1977; LeVine 2007; LeVine, Miller, and West 1988; Schwartzman 2001; Shweder et al. 2006; Super 1981; Werner 1988.

2 See, for example, Kendon 1997, 2004; Kita 2003, 2009; Kleinke 1986; Rossano, Brown, and Levinson 2009; Rutter 1984.

3 Kulick (1992: 195) claims that, when Gapun adults (mainland New Guinea) speak to infants and small children, they switch to Tok Pisin to secure and hold the child's attention. Gapun children learn Tok Pisin rather than the indigenous language, perhaps as an unintentional consequence of this caregiving practice.

4 For example, there are disagreements over the criteria for establishing that infants are indeed engaged in joint attention, and the integration of gaze with pointing (Franco and Butterworth 1996; Liskowski 2006; Masataka 2003b). There is also debate about the developmental sources of index-finger pointing. Vygotsky (1962) thought that it develops out of reaching and grasping movements. Masataka (2003a) argues that it develops from the three-to-four-month index-finger extensions of infants, and is shaped by interactants' responses to these behaviors.

5 We do not actually know whether pointing is universal; it certainly is subject to cultural variation (Kita 2003, 2009; Kendon 2004). If not, sharing of attention must be accomplished by other means. Even within developmentalist studies, there are individual differences in pointing that depend on interactional patterns. For example, pointing behavior is encouraged if mothers respond to early infant index-finger extensions by pointing themselves (Masataka 2003b).

6 This picture contrasts strongly with that described by Kulick (1992) for the Gapun of mainland New Guinea - the Gapun, like the Tzeltal Maya, hardly speak to infants under six months, indicating wide variation in interaction with infants even within geographical and cultural areas.

7 This research is part of an ongoing large comparative project on multimodal interaction at the Max Planck Institute for Psycholinguistics in the Netherlands, which explores universals in interactional organization and cultural differences in interactional style. Tzeltal and Rossel are also the focus of comparative study of adult interaction, which turns out to be quite different in some respects - for example, pace, feedback mechanisms, gaze behavior (Brown and Levinson 2005; Rossano, Brown, and Levinson 2009). Further research with Tzeltal and Rossel infants is being undertaken in collaboration with the Project Group on Communication before Language, headed by Ulf Liszkowski, at the Max Planck Institute.

8 'Primary focus of attention' was judged by gaze and other signals of attention focus; there were no cases of double coding (e.g. both $\mathrm{SO}$ and $\mathrm{PwO}$ ).

9 There is variation across infants, depending on the time of day of individual samples and the activities involved. Yet it is still clear that the pattern of social orientation (SO) is much higher for Rossel than for Tzeltal infants: for only one of the Tzeltal infants was social orientation the predominant activity sampled, while social orientation prevailed for all but two of the Rossel infants. 
10 The following were not considered to be new sequences: mere physical contact without vocalization and/or gaze, laughter, self-absorbed vocalizations (with no sign of intention to communicate), or immediate repeats of the same action.

11 The coding scheme was developed with the help of Suzanne Gaskins, to whom I am also indebted both for theoretical discussions and for advice about the logistics of studying infant interaction in field conditions. See also a related coding scheme in Bakeman and Adamson (1984).

12 Ball and soccer are English borrowings in Yélî Dnye. Ii and hii are attention-getters.

13 'To me' is a standard invitation to play ball together.

14 In the data analyzed so far, 11 of 20 Tzeltal infants (age range 11-15 months) and all nine Rossel infants (age range 10-15 months) pointed at least once during the periods of observation or were reported by their mothers to be pointing. A detailed quantitative comparison awaits the analysis of data from more controlled situations (Liskowski and Brown 2007).

15 A recent study of joint attention in a rural community in Nigeria (Childers, Vaughan, and Burquest 2007), comparable in some respects to the Tzeltal community, also found that by early in the second year the toddlers' joint attention behaviors did not differ from those of American toddlers, as reported, for example, in Bakeman and Adamson (1984).

\section{REFERENCES}

Bakeman, R. and Adamson, L. B. (1984) Coordinating attention to people and objects in mother-infant and peer-infant interactions. Child Development 55: 1278-89.

Bakeman, R., Adamson, L. B., Konner, M., and Barr, R. G. (1990) !Kung infancy: The social context of object exploration. Child Development 61: 794-809.

Bates, E., Benigni, L., Bretherton, I., Camaioni, L., and Volterra, V. (1979) The Emergence of Symbols: Cognition and Communication in Infancy. New York: Academic Press.

Bigelow, A. E. and Rochat, P. (2006)

Two-month-old infants' sensitivity to social contingency in mother-infant and stranger-infant interaction. Infancy 9(3): 313-25.

Brazelton, T. B. (1977) Implications of infant development among the Mayan Indians of Mexico. In P. H. Leiderman, S. R. Tulkin, and A. H. Rosenfeld (eds.), Culture and Infancy. 151-87. New York: Academic Press.
Brooks, R. and Meltzoff, A. N. (2008) Infant gaze following and pointing predict accelerated vocabulary growth through two years of age: A longitudinal growth curve modeling study. Journal of Child Language 35: 207-20.

Brown, P. (2007) The integration of gaze and pointing in infant-caregiver interaction in two cultures. Paper delivered at the 106th annual meetings of the American Anthropological Association. San Francisco, CA.

Brown, P. and Levinson, S. C. (2005) Comparative response systems. Paper delivered at the 104th annual meetings of the American Anthropological Association. Washington, DC.

Bruner, J. (1975a) The ontogenesis of speech acts. Journal of Child Language 2(1): 1-19.

Bruner, J. (1975b) From communication to language - A psychological perspective. Cognition 3(3): 255-87.

Bruner, J. (1982) Child's Talk. New York: Norton. 
Bruner, J. (1995) From joint attention to the meeting of minds: An introduction. In C. Moore and P. Dunham (eds.), Joint Attention: Its Origins and Role in Development. 1-14. Hillsdale, NJ:

Lawrence Erlbaum.

Bullowa, M. (ed.) (1979) Before Speech: The Beginning of Interpersonal Communication. Cambridge: Cambridge University Press. Butterworth, G. (2003) Pointing is the royal road to language for babies. In S. Kita (ed.), Pointing: Where Language, Culture, and Cognition Meet. 9-33. Mahwah, NJ: Lawrence Erlbaum.

Carpenter, M., Nagell, K., and Tomasello, M. (1998) Social cognition, joint attention, and communicative competence from 9 to 15 months of age. Monographs of the Society for Research in Child Development 63(4): Serial No. 255.

Chavajay, P. and Rogoff, B. (1999) Cultural variation in management of attention by children and their caregivers.

Developmental Psychology 35: 1079-90.

Childers, J. B., Vaughan, J. and Burquest, D. A. (2007) Joint attention and word learning in Ngas-speaking toddlers in Nigeria. Journal of Child Language 33: 199-225.

Clark, E. V. (2001) Grounding and attention in language acquisition. In M. Andronis, C., Ball, H. Elston, and S. Neuvel (eds.), Papers from the 37th meeting of the Chicago Linguistic Society, Vol. 1. 95-116. Chicago, IL: Chicago Linguistic Society.

Clark, H. H. (1996) Using Language. Cambridge: Cambridge University Press. de León, L. (1998) The emergent participant. Journal of Linguistic Anthropology 8(2): 131-61.

Eibl-Eibesfeld, I. (1983) Patterns of parent-child interaction in a crosscultural perspective. In A. Oliverio (ed.), The Behavior of Human Infants. 177-217. New York: Plenum Press.

Elman, J. L., Bates, E. A., Johnson, M. H., Karmiloff-Smith, A., Parisi, D., and
Plunkett, K. (1996) Rethinking Innateness:

A Connectionist Perspective on

Development. Cambridge, MA: MIT

Press/Bradford Books.

Estigarribia, B. and Clark, E. V. (2007) Getting and maintaining attention in talk to young children. Journal of Child Language 34: 799-814.

Evans, N. and Levinson, S. C. (2009) The myth of language universals: Language diversity and its importance for cognitive science. Behavioral and Brain Sciences 32(5): 429-92.

Farroni, T., Csibra, G., Simion, F. F., and Johnson, M. H. (2002) Eye contact detection in humans from birth. Proceedings of the National Academy of Sciences of the United States of America 99: 9602-5.

Fernald, A. (1992a) Meaningful melodies in mothers' speech to infants. In H. Papoušek, U. Jürgens, and M. Papoušek (eds.), Nonverbal Vocal Communication: Comparative and Developmental Approaches. 262-82. Cambridge: Cambridge University Press.

Fernald, A. (1992b) Human maternal vocalizations to infants as biologically relevant signals: An evolutionary perspective. In J. H. Barkow, L. Cosmides, and J. Tooby (eds.), The Adapted Mind. 391-428. New York: Oxford University Press.

Field, T. M., Sostek, A. M., Vietze, P., and Leiderman, P. H. (1981) Culture and Early Interactions. Hillsdale, NJ: Lawrence Erlbaum.

Franco, F. and Butterworth, G. (1996) Pointing and social awareness: Declaring and requesting in the second year. Journal of Child Language 23: 307-36.

Gratier, M. (2003) Expressive timing and interactional synchrony between mothers and infants: Cultural similarities, cultural differences, and the immigration experience. Cognitive Development 18: 533-54.

Gratier, M. and Trevarthen, C. (2008) Musical narrative and motives for 
culture in mother-infant vocal interaction. Journal of Consciousness Studies 15(10-11): 122-58.

Harkness, S. and Super, C. (eds.) (1996) Parents' Cultural Belief Systems: Their Origins, Expressions, and Consequences. New York: The Guilford Press.

Jahoda, G. and Lewis, I. M. (1988) Acquiring Culture: Cross-Cultural Studies in Child Development. London: Croom Helm.

Jones, S. and Zimmerman, D. (2003) A child's point and the achievement of intentionality. Gesture 3: 155-85.

Keenan, E. O. and Schieffelin, B. (1976) Topic as a discourse notion: A study of topic in the conversations of children and adults. In C. Li (ed.), Subject and Topic. 335-84. New York: Academic Press.

Kelly, S., Manning, S. M., and Rodak, S. (2008) Gesture gives a hand to language and learning: Perspectives from cognitive neuroscience, developmental psychology and education. Language and Linguistics Compass 2/4: 569-88.

Kendon, A. (1997) Gesture. Annual Review of Anthropology 26: 109-28.

Kendon, A. (2004) On pointing. In A. Kendon (ed.), Gesture: Visible Action as Utterance. 199-224. Cambridge: Cambridge University Press.

Kidwell, M. (2003) 'Looking to See if Someone is Looking at You': Gaze and the Organization of Observability in Very Young Children's Harassing Acts Toward a Peer. Doctoral Dissertation. Santa Barbara, CA: University of California.

Kidwell, M. (2005) Gaze as social control: How very young children differentiate 'the look' from a 'mere look' by their adult caregivers. Research on Language and Social Interaction 38(4): 417-49.

Kidwell, M. (2009) Gaze shift as an interactional resource for very young children. Discourse Processes 46: 145-60.

Kidwell, M. and Zimmerman, D. (2006) 'Observability' in the interactions of very young children. Communication Monographs 73(1): 1-28.
Kidwell, M. and Zimmerman, D. (2007) Joint attention as action. Journal of Pragmatics 39: 592-611.

Kita, S. (ed.) (2003) Pointing: Where Language, Culture, and Cognition Meet. Mahwah, NJ: Lawrence Erlbaum.

Kita, S. (2009) Cross-cultural variation of speech-accompanying gesture: A review. Language and Cognitive Processes 24(2): 145-67.

Kleinke, C. L. (1986) Gaze and eye contact: A research review. Psychological Bulletin 100(1): 78-100.

Kuhl, P. K., Andruski, J., Chistovich, I., Chistovich, L., Kozhevnikova, E., Ryskina, V., Stolyarova, E., Sundberg, U., and Lacerda, F. (1997) Cross-language analysis of phonetic units in language addressed to infants. Science 277: 684-6.

Kulick, D. (1992) Language Shift and Cultural Reproduction: Socialization, Self, and Syncretism in a Papua New Guinean Village. Cambridge: Cambridge University Press.

Lancy, D. F. (1996) Playing on the Mother-Ground: Cultural Routines for Children's Development. New York: Guilford Press.

Lancy, D. F. (2008) The Anthropology of Childhood: Cherubs, Chattels, Changelings. Cambridge: Cambridge University Press.

Leiderman, H. P., Tulkin, S. R., and Rosenfeld, A. H. (eds.) (1977) Culture and Infancy: Variations in the Human Experience. New York: Academic Press.

LeVine, R. (2007) Ethnographic studies of childhood: A historical overview. American Anthropologist 109(2): 247-60.

LeVine, R. A., Miller, P. M., and West, M. M. (eds.) (1988) Parental Behavior in Diverse Societies. San Francisco, CA: Jossey-Bass Inc.

LeVine, R., Dixon, S. LeVine, S., Richman, A., Liederman, P. H., Keefer, C. H., and Brazelton, T. B. (1994) Child Care and Culture: Lessons From Africa. New York: Cambridge University Press. 
Levinson, S. C. (2006) On the human 'interaction engine.' In N. Enfield and S. C. Levinson (eds.), Roots of Sociality: Culture, Cognition, and Interaction. 153-78. Oxford: Berg.

Liszkowski, U. (2005) Human twelvemonth-olds point cooperatively to share interest with and helpfully provide information for a communicative partner. Gesture 5(1-2): 135-54.

Liszkowski, U. (2006) Infant pointing at twelve months: Communicative goals, motives, and social-cognitive abilities. In N. Enfield and S. C. Levinson (eds.), Roots Of Sociality: Culture, Cognition, and Interaction. 153-78. Oxford: Berg.

Liszkowski, U. and Brown, P. (2007) Infant pointing (9 to 15 months) in different cultures. In A. Majid (ed.), Field Manual Volume 10. 82-9. Nijmegen, The Netherlands: Max Planck Institute for Psycholinguistics.

Liszkowski, U., Brown, P., Callaghan, T., Takada, A., and de Voss, C. (forthcoming) A gestural prelinguistic universal of human communication.

Liszkowski, U., Carpenter, M., Henning, A., Striano, T., and Tomasello, M. (2004) Twelve-month-olds point to share attention and interest. Developmental Science 7(3): 297-307.

Liszkowski, U., Carpenter, M., and Tomasello, M. (2007) Pointing out new news, old news, and absent referents at 12 months of age. Developmental Science 10(2): F1-F7.

Liszkowski, U., Carpenter, M., and

Tomasello, M. (2008) Twelve-month-olds communicate helpfully and appropriately for knowledgeable and ignorant partners. Cognition 108(3): 732-9.

Liszkowski, U., Schäfer, M., Carpenter, M., and Tomasello, M. (2009) Prelinguistic infants, but not chimpanzees, communicate about absent entities. Psychological Science 20: 654-60.

Masataka, N. (2003a) From index-finger extension to index-finger pointing:
Ontogenesis of pointing in preverbal infants. In Sotaro Kita (ed.), Pointing: Where Language, Culture and Cognition Meet. 68-84. Mahwah, NJ: Lawrence Erlbaum.

Masataka, N. (2003b) The Onset of Language. Cambridge: Cambridge University Press.

Meltzoff, A. N. and Moore, M. K. (1977) Imitation of facial and manual gestures by human neonates. Science 198(4312): 75-8.

Monnot, M. (1999) Function of infantdirected speech. Human Nature 10: 415-43.

Moore, C. and Dunham, P. J. (eds.) (1995) Joint Attention: Its Origins and Role in Development. Hillsdale, NJ: Lawrence Erlbaum.

Murray, L. and Trevarthen, C. (1985) Emotional regulation of interactions between two-month-olds and their mothers. In T. M. Field and N. A. Fox (eds.), Social Perception in Infants. 177-97. Norwood, NJ: Ablex.

Ninio, A. and Bruner, J. (1978) The achievement and antecedents of labelling. Journal of Child Language 5: 1-15.

Ochs, E. (1988) Culture and Language Development. Cambridge: Cambridge University Press.

Ochs, E. and Schieffelin, B. B. (1984) Language acquisition and socialization: Three developmental stories and their implications. In R. A. Shweder and R. LeVine (eds.), Culture Theory: Essays on Mind, Self, and Emotion. 276-320. New York: Cambridge University Press.

Paradise, R. and Rogoff, B. (2009) Side by side: Learning by observing and pitching in. Ethos 37(1): 102-38.

Rogoff, B., Mistry, J., Göncü, A., and Mosier, C. (1993) Guided participation in cultural activity by toddlers and caregivers. Monographs of the Society for Research in Child Development 58(8).

Rogoff, B., Paradise, R., Arauz, R. M., Correa-Chavez, M., and Angelillo, C. 
(2003) Firsthand learning through intent participation. Annual Review of Psychology 54: 175-203.

Rossano, F. (2009) Gaze Behavior in Face to Face Interactions. Doctoral Dissertation. Nijmegen, The Netherlands: Radboud University.

Rossano, F., Brown, P., and Levinson, S. C. (2009) Gaze and questioning in three cultures. In J. Sidnell (ed.), Comparative Studies in Conversation Analysis. 187-249. Cambridge: Cambridge University Press.

Rutter, D. R. (1984) Looking and Seeing: The Role of Visual Communication in Social Interaction. Chichester, U. K. and New York: Wiley.

Schieffelin, B. B. (1990) The Give and Take of Everyday Life: Language Socialization of Kaluli Children. Cambridge: Cambridge University Press.

Schieffelin, B. B. and Ochs, E. (1983) A cultural perspective on the transition from prelingusitic to linguistic communication. In R. Golinkoff (ed.), The Transition From Prelinguistic to Linguistic Communication. 115-31. Hillsdale NJ: Lawrence Erlbaum.

Schieffelin, B. B. and Ochs, E. (1986) Language Socialization Across Cultures. Cambridge: Cambridge University Press.

Schwartzman, H. (2001) Children and anthropology: A century of studies. In H. Schwartzman (ed.), Children and Anthropology: Perspectives for the 21st Century. 15-37. Westport, CT: Bergin and Garvey.

Shweder, R. A., Goodnow, J. J., Hatano, G., LeVine, R. A., Marcus, H. R., and Miller, P. J. (2006) The cultural psychology of development: One mind, many mentalities. In W. Damon and R. M. Lerner (eds.), Handbook of Child Psychology, Vol. 1. 6th ed. 716-92. New York: John Wiley and Son.

Stivers, T., Enfield, N. J., Brown, P., Englert, C., Hayashi, M., Heinemann, T., Hoymann, G., Rossano, F., de Ruiter, J.-P., Yoon, K.-E., and Levinson, S. C.
(2009) Universals and cultural variation in turn-taking in conversation.

Proceedings of the National Academy of Sciences of the United States of America 106: 10587-10592.

Super, C. M. (1981) Cross-cultural research on infancy. In H. C. Triandis and A. Heron (eds.), Handbook of Cross-Cultural Psychology, Vol. 4: Developmental Psychology. 17-53. Boston, MA: Allyn and Bacon.

Tomasello, M. (1999) The Cultural Origins of Human Cognition. Cambridge, MA: Harvard University Press.

Tomasello, M. (2008) Origins of Human Communication. Cambridge, MA: MIT Press.

Tomasello, M., Carpenter, M., Call, J., Behne, T., and Moll, H. (2005) Understanding and sharing intentions: The origins of cultural cognition. Behavioral and Brain Sciences 28: 675-91.

Tomasello, M., Carpenter, M., and Liszkowski, U. (2007) A new look at infant pointing. Child Development 78(3): 705-22.

Tomasello, M. and Ferrar, M. J. (1986) Joint attention and early language. Child Development 57: 1454-63.

Tomasello, M. and Todd, J. (1983) Joint attention and lexical acquisition style. First Language 4: 197-211.

Trevarthen, C. (1988) Universal cooperative motives: How infants begin to know the language and culture of their parents. In G. Jahoda and I. Lewis (eds.), Acquiring Culture: Cross-Cultural Studies in Child Development. 37-90. London: Croon Helm.

Vygotsky, L. (1962) Thought and Language. Boston, MA: MIT Press.

Werner, E. E. (1988) A cross-cultural perspective on infancy. Journal of Cross-Cultural Psychology 19: 96-113.

Wootten, A. J. (1997) Interaction and the Development of Mind. Cambridge: Cambridge University Press. 